\title{
Neutrosophic Generalized Regular Contra Continuity in Neutrosophic Topological Spaces
}

\author{
Blessie Rebecca. $S^{1} \quad$ A. Francina Shalini ${ }^{2}$ \\ 1. PG Scholar, Department of Mathematics,Nirmala College for women, Coimbatore, Tamilnadu, India \\ Email-blessie.rebecca96@gmail.com \\ 2. Assistant Professor, Department of Mathematics,Nirmala College for women, Coimbatore, Tamilnadu, India.
}

\begin{abstract}
In this paper, the concept of Neutrosophic generalized regular contra continuous mapping are introduced. Furthermore Neutrosophic generalized regular contra irresolute mapping, Strongly Neutrosophic generalized regular contra continuous mapping and Perfectly Neutrosophic generalized regular contra continuous mapping are introduced in Neutrosophic topological spaces.
\end{abstract}

Index terms- Neutrosophic generalized regular contra continuity; Neutrosophic generalized regular contra irresolute; Strongly Neutrosophic generalized regular contra continuity and Perfectly Neutrosophic generalized regular contra continuity.

\section{INTRODUCTION}

Topology is a classical subject, as a generalization of topological spaces many type of topological spaces introduced over the year. C.L.Chang[6] introduced and developed fuzzy topological space by using L.A. Zadeh[19] fuzzy sets. Coker[7] introduced the notion of Intuitionistic fuzzy topological spaces by using Atanassov[3] intuitionistic fuzzy set .Neutrality the degree of indeterminacy, as an independent concept, was introduced by Smarandache[13] He also defined the Neutrosophic set on three component Neutrosophic topological spaces (t,f,i) =(Truth, Falsehood, Indeterminacy). The Neutrosophic crisp set concept was converted to Neutrosophic topological spaces by A.A.Salama[16]. R.Dhavaseelan[8] introduced Neutrosophic generalized closed sets. V.K.Shanthi[18] introduced Neutrosophic Generalized Semi Closed Sets In Neutrosophic Topological Spaces. A.A.Salama[17] introduced Neutrosophic Closed Set and Neutrosophic Continuous Functions. R.Dhavaseelan [11] investigated Generalized intuitionistic fuzzy contracontinuous functions and Dontchev [12] studied the concept of Contra continuous functions and strongly Sclosed spaces. R. Dhavaseelan [9] introduced the concept of Neutrosophic generalized $\alpha$ contracontinuity.

\section{PRELIMINARIES}

Definition 2.1 $[8,9]$

Let $X$ be a non-empty fixed set. A Neutrosophic set $A$ has the form

$A=\left\{\left(x, \mu_{A}(x), \sigma_{A}(x), \gamma_{A}(x)\right): x \in X\right\}$

where $\mu_{A}(x), \sigma_{A}(x), \gamma_{A}(x)$ are topological spaces and $\mu_{A}(x)$ is the degree of membership function,

$\sigma_{A}(x)$ is the degree of indeterminacy and $\gamma_{A}(x)$ is the degree of non-membership function respectively of each $x \in X$ to the set $A$.

\section{Remark 2.2 [8,9]}

A Neutrosophic set $A=\left\{\left(x, \mu_{A}(x), \sigma_{A}(x), \gamma_{A}(x)\right): x \in\right.$ $X$ \} can be identified to an ordered triple $\left(\mu_{A}, \sigma_{A}, \gamma_{A}\right)$ in $] 0^{-}, 1^{+}$[on $X$.

\section{Example 2.3 [8,9]}

Since our main purpose is to construct the tools for developing Neutrosophic set and Neutrosophic topology, we must introduce the Neutrosophic set $0_{N}$ and $1_{N}$ in $X$ as follows:

$0_{N}=\{(x, 0,0,1): x \in X\}$

$1_{N}=\{(x, 1,0,0): x \in X\}$

\section{Definition 2.4 [4]}

Let $A=\left\{\left(x, \mu_{A}, \sigma_{A}, \gamma_{A}\right)\right\}$ be Neutrosophic set on $X$ , then Complement of set $A$ i.e., $A^{C}$ is defined as $A^{C}=\left\{\left(x, \gamma_{A}(x), 1-\sigma_{A}(x), \mu_{A}(x)\right): x \in X\right\}$.

Definition 2.5 [8,9]

Let $X$ be a non-empty set and $A$ and $B$ are Neutrosophic sets of the form

$A=\left\{\left(x, \mu_{A}(x), \sigma_{A}(x), \gamma_{A}(x)\right): x \in X\right\}$ and

$\mathrm{B}=\left\{\left(\mathrm{x}, \mu_{\mathrm{B}}(\mathrm{x}), \sigma_{\mathrm{B}}(\mathrm{x}), \gamma_{\mathrm{B}}(\mathrm{x})\right): \mathrm{x} \in \mathrm{X}\right\}$ then we consider the definition of subset $(A \subseteq B)$ is defined as $A \subseteq B \Leftrightarrow \mu_{A}(x) \leq \mu_{B}(x), \sigma_{A}(x) \leq \sigma_{B}(x), \gamma_{A}(x) \geq \gamma_{B}(x)$, for all $x \in X$.

\section{Theorem 2.6 [4]}

For any Neutrosophic set $A$ the following condition holds

(i) $0_{N} \subseteq A, 0_{N} \subseteq 0_{N}$,

(ii) $A \subseteq 1_{N}, 1_{N} \subseteq 1_{N}$.

Definition 2.7 [8,9]

Let $X$ be a non-empty set and

$A=\left\{x, \mu_{A}(x), \sigma_{A}(x), \gamma_{A}(x)\right\}$ and 
$B=\left\{x, \mu_{B}(x), \sigma_{B}(x), \gamma_{B}(x)\right\}$ are Neutrosophic sets then $A \cap B$ is defined as

$A \cap B=\left\{x, \mu_{A}(x) \wedge \mu_{B}(x), \sigma_{A}(x) \wedge \sigma_{B}(x), \gamma_{A}(x) \vee\right.$ $\left.\gamma_{B}(x)\right\}$

then $A \cup B$ is defined as

$A \cup B=\left\{x, \mu_{A}(x) \vee \mu_{B}(x), \sigma_{A}(x) \vee \sigma_{B}(x), \gamma_{A}(x) \wedge\right.$ $\left.\gamma_{B}(x)\right\}$.

\section{Definition 2.8 [8,9]}

A Neutrosophic topology is a non-empty set $X$ is an family $\tau_{N}$ of Neutrosophic subsets in $X$ satisfying the axioms:

(i) $0_{N}, 1_{N} \in \tau_{N}$

(ii) $G_{1} \cap G_{2} \in \tau_{N}$ for any $G_{1}, G_{2} \in \tau_{N}$

(iii) $\cup G_{i} \in \tau_{N}$ for every $\left\{G_{j}: \mathrm{j} \in \mathrm{J}\right\} \subseteq \tau_{N}$

the pair $\left(X, \tau_{N}\right)$ is called Neutrosophic topological space.

The element in Neutrosophic topological space $\left(X, \tau_{N}\right)$ are called Neutrosophic open sets.

A Neutrosophic set $F$ is closed if and only if $(F)^{C}$ is Neutrosophic open.

Definition 2.9 [8,9]

Let $\left(X, \tau_{N}\right)$ Neutrosophic topological spaces and $A=\left\{\left(x, \mu_{A}(x), \quad \sigma_{A}(x), \quad \gamma_{A}(x)\right): x \quad x \in X\right\}$ be Neutrosophic set in $X$. Then the

Neutrosophic closure and Neutrosophic interior are defined as

$\operatorname{Ncl}(A)=\bigcap\{\mathrm{K}: \mathrm{K}$ is Neutrosophic closed set in $X$ and $A \subseteq K\}$,

$\operatorname{Nint}(A)=\bigcup\{\mathrm{G}: \mathrm{G}$ is Neutrosophic open set in $X$ and $G \subseteq A\}$.

\section{Definition 2.10 [4]}

$A$ is Neutrosophic open set if and only if

$\mathrm{A}=\operatorname{Nint}(A)$,

$A$ is Neutrosophic closed set if and only if

$\mathrm{A}=\operatorname{Ncl}(A)$.

\section{Theorem 2.11 [4]}

Let $\left(X, \tau_{N}\right)$ Neutrosophic topological spaces and $A, B$ be two Neutrosophic sets in $X$. Then the following properties holds:

(i) $A \subseteq B \Rightarrow \operatorname{Nint}(A) \subseteq \operatorname{Nint}(B)$,

(ii) $A \subseteq B \Rightarrow \operatorname{Ncl}(A) \subseteq N \operatorname{Nol}(B)$,

(iii) $\operatorname{Nint}(\operatorname{Nint}(A))=\operatorname{Nint}(A)$,

(iv) $\operatorname{Ncl}(\operatorname{Ncl}(A))=\operatorname{Ncl}(A)$,

(v) $\operatorname{Nint}(A \cap B)=\operatorname{Nint}(A) \cap \operatorname{Nint}(B)$,

(vi) $\operatorname{Ncl}(A \cup B)=\operatorname{Ncl}(A) \cup \operatorname{Ncl}(B)$,

(vii) $\operatorname{Nint}\left(O_{N}\right)=O_{N}$,

(viii) $\operatorname{Nint}\left(1_{N}\right)=1_{N}$,

(ix) $\mathrm{Ncl}\left(\mathrm{O}_{N}\right)=O_{N}$,

(x) $\operatorname{Ncl}\left(1_{N}\right)=1_{N}$,

(xi) $\operatorname{Ncl}(A \cap B) \subseteq N c l(A) \cap N \operatorname{Nol}(B)$,

(xii) $\operatorname{Nint}(A \cup B) \supseteq \operatorname{Nint}(A) \cup \operatorname{Nint}(B)$.

Definition 2.12 [2,4]
A subset $A$ of Neutrosophic space $\left(X, \tau_{N}\right)$ is called Neutrosophic regular open (in short NR open) if $A=\operatorname{Nint}(N \operatorname{Ncl}(A))$.The Complement of NR open set is called NR closed.

Definition 2.13 [4]

A subset $A$ of Neutrosophic space $\left(X, \tau_{N}\right)$ is called Neutrosophic generalized closed (in short $N G$ closed ) if $\operatorname{Ncl}(A) \subseteq U$, whenever $A \subseteq U$ and $U$ is Neutrosophic open. The Complement of a NG closed set is called NG open set.

\section{Definition 2.14 [4]}

Let $A$ be a subset of Neutrosophic space $\left(X, \tau_{N}\right)$ is called Neutrosophic generalized regular closed (NGR closed) if Neutrosophic Regular cl $(A) \subseteq U$

(in short $\operatorname{NRcl}(A) \subseteq U$ ), whenever $A \subseteq U$ and $U$ is Neutrosophic open.The Complement of a NGR closed set is called NGR open set.

\section{Definition 2.15 [5]}

Let $(X, T)$ and $(Y, S)$ be any two Neutrosophic topological spaces

(i) A map $f:(X, T) \rightarrow(Y, S)$ is said to be Neutrosophic continuous if the inverse image of every Neutrosophic closed set in $(Y, S)$ is Neutrosophic closed set in $(X, T)$.

\section{Definition 2.16 [5]}

Let $(X, T)$ and $(Y, S)$ be any two Neutrosophic topological spaces

(i) A map $f:(X, T) \rightarrow(Y, S)$ is said to be Neutrosophic generalized regular continuous (in short NGR continuous) if the inverse image of every Neutrosophic closed set in $(Y, S)$ is NGR closed set in $(X, T)$.

(ii) A map $f:(X, T) \rightarrow(Y, S)$ is said to be Neutrosophic generalized regular irresolute (in short $N G R$ irresolute) if the inverse image of every NGR closed set in $(Y, S)$ is NGR closed set in $(X, T)$.

(iii) A map $f:(X, T) \rightarrow(Y, S)$ is said to be Strongly Neutrosophic generalized regular continuous (in short Strongly NGR continuous) if the inverse image of every NGR open set in $(Y, S)$ is an Neutrosophic open set in $(X, T)$.

Definition 2.17 [9]

Let $(X, T)$ and $(Y, S)$ be any two neutrosophic topological spaces.

(i) A function $f:(X, T) \rightarrow(Y, S)$ is called Neutrosophic contra-continuous if the inverse image of every Neutrosophic open set in $(Y, S)$ is a Neutrosophic closed set in $(X, T)$. 
(ii) A function $f:(X, T) \rightarrow(Y, S)$ is called Neutrosophic generalized contra- continuous (in short $N G$ contra-continuous) if the inverse image of every Neutrosophic open set in $(Y, S)$ is a $N G$ closed set in $(X, T)$.

\section{NEUTROSOPHIC GENERALIZED REGULAR CONTRA CONTINUITY Definition 3.1}

Let $(X, T)$ and $(Y, S)$ be any two Neutrosophic topological spaces.

(i) A function $f:(X, T) \rightarrow(Y, S)$ is said to be Neutrosophic regular contra-continuous (in short $N R$ contra-continuous) if the inverse image of every Neutrosophic open set in $(Y, S)$ is $N R$ closed set in $(X, T)$.

(ii) A function $f:(X, T) \rightarrow(Y, S)$ is called a Neutrosophic generalized regular contracontinuous (in short NGR contra-continuous) if $f$ ${ }^{-1}(B)$ is a NGR closed set in $(X, T)$ for everyNeutrosophic open set $B$ in $(Y, S)$.

(iii) A function $f:(X, T) \rightarrow(Y, S)$ is called a Strongly Neutrosophic generalized regular contracontinuous (in short Strongly NGR contracontinuous) if $f^{-1}(B)$ is a Neutrosophic closed set in $(X, T)$ for every NGR open set $B$ in $(Y, S)$.

(iv) A function $f:(X, T) \rightarrow(Y, S)$ is called a Neutrosophic generalized regular contra irresolute (in short NGR contra irresolute), if $f^{-1}(B)$ is a $N G R$ closed set in $(X, T)$ for every NGR open set $B$ in $(Y, S)$.

\section{Theorem 3.2}

Let $(X, T)$ and $(Y, S)$ be any two Neutrosophic topological spaces. If $f:(X, T) \rightarrow(Y, S)$ be Neutrosophic contra-continuous function. Then it is a NGR contra-continuous mapping.

Proof:

Let $B$ be a Neutrosophic open sets in $(Y, S)$

Since $f$ is Neutrosophic contra-continuous function ,

By definition 2.17(i) , $f^{-1}(B)$ is Neutrosophic closed set in $(X, T)$.

We know, Every Neutrosophic closed set is NGR closed set.

Now $f^{-1}(B)$ is NGR closed set in $(X, T)$.

Therefore By definition 3.1(ii), $f$ is NGR contracontinuous mapping. Hence proved.

The converse of theorem 3.2 need not be true as shown in example 3.2.1

Example 3.2.1

Let $X=\{a, b\}$ and where $A=\{(0.6,0.6,0.6),(0.4$, $0.4,0.4)\}$ and $B=\{(0.2,0.2,0.3),(0.8,0.8,0.7)\}$ is
Neutrosophic sets. Then the families $T=\left\{0_{N}, 1_{N}\right.$, $A\}$ and $S=\left\{0_{N}, 1_{N}, B\right\}$ are Neutrosophic topologies on $\mathrm{X}$. Define a function $f:(X, T) \rightarrow$ $(Y, S)$ as $f(a)=a, f(b)=b$

Then $f$ is a NGR contra-continuous function but $f$ ${ }^{-1}(B)$ is not a Neutrosophic closed set in $(X, T)$.

Hence $f$ is not a Neutrosophic contra-continuous function.

\section{Theorem 3.3}

Every NR closed sets is NGR closed set. But the converse is not true.

Proof :

Let $A$ be $N R$ closed sets in $X$ and let $A \subseteq U$ and $U$ is Neutrosophic open set in $X$.

We know that $A=\operatorname{Ncl}(\operatorname{Nint}(A))$ (By definition 2.12)

$\Rightarrow \operatorname{Ncl}(\operatorname{Nint}(A)) \subseteq U$

That is $\operatorname{NRcl}(A) \subseteq U$

Therefore we have $\operatorname{NRcl}(A) \subseteq U$, whenever $A \subseteq U$ and $U$ is Neutrosophic open set in $X$.

Therefore by definition $2.14, A$ is NGR closed set in $X$. Hence proved.

The converse of theorem 3.3 is not true is shown in example 3.4

\section{Example 3.4}

Let $X=\{a, b\}$ and where $A_{1}=\{(0.4,0.6,0.5),(0.7$, $0.3,0.6)\}$,

$A_{2}=\{(0.3,0.6,0.8),(0.6,0.3,0.6)\}$ and $\tau_{N}=\left\{0_{N}\right.$, $\left.A_{1}, A_{2}, 1_{N}\right\}$ is Neutrosophic topological space. Then $A_{3}=\{(0.5,0.7,0.5),(0.9,0.4,0.5)\}$ is NGR closed set but not NR closed set.

\section{Theorem 3.5}

Let $(X, T)$ and $(Y, S)$ be any two Neutrosophic topological spaces.

If $f:(X, T) \rightarrow(Y, S)$ is a NR contra-continuous function then $f$ is a NGR contra-continuous function.

Proof:

Let $B$ be a Neutrosophic open set in $(Y, S)$.

Since $f$ is a NR contra-continuous function,

By definition 3.1(i) $f^{-1}(B)$ is a $N R$ closed set in $(X, T)$.

Since by theorem 3.3, We know, Every NR closed set is a $N G R$ closed set.

We get, $f^{-1}(B)$ is a $N G R$ closed set in $(X, T)$.

Hence by definition 3.1(ii), $f$ is a NGR contracontinuous function. Hence proved.

The converse of theorem 3.5 need not be true as shown in example 3.5.1.

\section{Example 3.5.1}

Let $X=\{a, b\}$ and where $A=\{(0.5,0.5,0.5),(0.5$, $0.5,0.5)\}$ and $B=\{(0.4,0.4,0.4),(0.6,0.6,0.6)\}$ is Neutrosophic sets. Then the families $T=\left\{0_{N}, 1_{N}\right.$ , $A\}$ and $S=\left\{0_{N}, 1_{N}, B\right\}$ are Neutrosophic topologies 
on X. Define a function $f:(X, T) \rightarrow(Y, S)$ as $f$ (a) $=a, f(b)=b$

Then $f$ is a NGR contra-continuous function but $f$ ${ }^{-1}(B)$ is not a NR closed set in $(X, T)$. Hence $f$ not a $N R$ contra-continuous function.

\section{Theorem 3.6}

For any two Neutrosophic topological spaces $(X, T)$ and $(Y, S)$. If $f:(X, T) \rightarrow(Y, S)$ is a Strongly $N G R$ contra-continuous function then $f$ is a NGR contra-continuous function.

Proof:

Let $B$ be a Neutrosophic open set in $(Y, S)$.

We know, Every Neutrosophic open set is a NGR open set.

Now, $B$ is a NGR open set in $(Y, S)$.

Since $f$ is a Strongly NGR contra-continuous function,

By definition 3.1(iii), $f^{-1}(B)$ is a Neutrosophic closed set in $(X, T)$.

Since every Neutrosophic closed set is a NGR closed set

\section{$f^{-1}(B)$ is a NGR closed set in $(X, T)$.}

Hence by definition 3.1(ii), $f$ is a NGR contracontinuous function.

Hence proved.

The converse of theorem 3.6 need not be true as shown in example 3.6.1.

\section{Example 3.6.1}

Let $X=\{a, b\}$ and where $A=\{(0.4,0.4,0.4),(0.3$, $0.3,0.3)\}$ and $B=\{(0.2,0.2,0.3),(0.8,0.8,0.7)\}$ is Neutrosophic sets. Then the families $T=\left\{0_{N}, 1_{N}\right.$, $A\}$ and $S=\left\{0_{N}, 1_{N}, B\right\}$ are Neutrosophic topologies on X. Define a function $f:(X, T) \rightarrow$ $(Y, S)$ as $f(a)=a, f(b)=b$. Then $f$ is a $N G R$ contra-continuous function. Let $C=\{(0.4,0.4,0.4)$, $(0.6,0.6,0.6)\}$ be a NGR open in $(X, T)$ set, but $f^{-1}(C)$ is not a Neutrosophic closed set in $(X, T)$. Hence $f$ is not a Strongly NGR contra-continuous function.

\section{Theorem 3.7}

For any two Neutrosophic topological spaces $(X, T)$ and $(Y, S)$. If $f:(X, T) \rightarrow(Y, S)$ is a Strongly $N G R$ contra-continuous function then $f$ is a Neutrosophic contra-continuous function.

Proof:

Let $B$ be a Neutrosophic open set in $(Y, S)$.

We know, Every Neutrosophic open set is a $N G R$ open set.

Now, $B$ is a NGR open set in $(Y, S)$.

Since $f$ is a Strongly NGR contra-continuous function,

By definition 3.1(iii), $f^{-1}(B)$ is a Neutrosophic closed set in $(X, T)$.

Hence by definition 2.17 (i), $f$ is a Neutrosophic contra-continuous function.

Hence proved.

\section{Theorem 3.8}

Let $(X, T),(Y, S)$ and $(Z, R)$ be any three Neutrosophic topological spaces. If a function

$f:(X, T) \rightarrow(Y, S)$ is strongly NGR continuous function and $g:(Y, S) \rightarrow(Z, R)$ is a NGR contracontinuous function then $g \circ f$ is a Neutrosophic contra-continuous function.

Proof:

Let $B$ be a Neutrosophic open set in $(Z, R)$.

Since $g$ is a NGR contra-continuous function,

By definition 3.1(ii), $g^{-1}(B)$ is NGR closed set in (Y, S).

Since $f$ is Strongly NGR continuous function,

By definition 2.16(iii),$f^{-1}\left(g^{-1}(B)\right)$ is a Neutrosophic closed set in $(X, T)$.

Hence by definition 2.17(i), $g \circ f$ is a Neutrosophic contra-continuous function. Hence proved.

\section{Theorem 3.9}

Let $(X, T),(Y, S)$ and $(Z, R)$ be any three Neutrosophic topological spaces. If $f$ is a NGR contra-continuous function and $g$ is a Neutrosophic continuous function, then $g \circ f$ is a NGR contra-continuous function.

Proof:

Let $B$ be a Neutrosophic open set in $(Z, R)$.

Since $g$ is a Neutrosophic continuous function,

By definition 2.15(i) $g^{-1}(B)$ is Neutrosophic open set in $(Y, S)$.

Since $f$ is a NGR contra-continuous function,

By definition 3.1(ii), $f^{-1}\left(g^{-1}(B)\right)$ is a NGR closed set in $(X, T)$.

Hence by definition 4.1(ii), $g \circ f$ is a NGR contracontinuous function. Hence proved.

\section{Theorem 3.10}

Let $(X, T),(Y, S)$ and $(Z, R)$ be any three Neutrosophic topological spaces. If $f$ is a NGR contra-continuous function and $g$ is a Neutrosophic contra-continuous function, then $g \circ f$ is a NGR continuous function.

Proof:

Let $B$ be a Neutrosophic open set in $(Z, R)$.

Since $g$ is a Neutrosophic contra- continuous function,

By definition 2.17(i),$g^{-1}(B)$ is Neutrosophic closed set in $(Y, S)$.

Since $f$ is a NGR contra-continuous function,

By definition 3.1(ii), $f^{-1}\left(g^{-1}(B)\right)$ is a NGR open set in $(X, T)$.

Hence by definition 3.1(i), $g \circ f$ is a NGR continuous function. Hence proved.

\section{Theorem 3.11}

Let $(X, T),(Y, S)$ and $(Z, R)$ be any three Neutrosophic topological spaces. If $f$ is a NGR contra-irresolute function and $g$ is a NGR contra-continuous function, then $g \circ f$ is a NGR continuous function. 
Proof:

Let $B$ be a Neutrosophic open set in $(Z, R)$.

Since $g$ is a NGR contra-continuous function, By definition 3.1(ii) $g^{-1}(B)$ is NGR closed set in ( $Y$, S).

Since $f$ is a NGR contra-irresolute function, By definition 3.1(iv), $f^{-1}\left(g^{-1}(B)\right)$ is a NGR open set in $(X, T)$.

Hence by definition 2.16(i), $g \circ f$ is a $N G R$ continuous function. Hence proved.

\section{Theorem 3.12}

Let $(X, T),(Y, S)$ and $(Z, R)$ be any three Neutrosophic topological spaces. If $f$ is a NGR irresolute function and

$g$ is a NGR contra-continuous function, then $g \circ f$ is a NGR contra-continuous function.

Proof:

Let $B$ be a Neutrosophic open set in $(Z, R)$.

Since $g$ is a NGR contra-continuous function,

By definition 3.1(ii) $g^{-1}(B)$ is $N G R$ closed set in $(Y$, $S)$.

Since $f$ is a NGR irresolute function,

By definition 2.16(ii), $f^{-1}\left(g^{-1}(B)\right)$ is a NGR closed set in $(X, T)$.

Hence by definition 3.1(ii), $g \circ f$ is a NGR contracontinuous function. Hence proved.

\section{REFERENCES}

[1] R. Anitha, Dr. D. Jayanthi, "Intuitionistic Fuzzy Regular Generalized Semi Continuous Mappings",International Journal of Science and Research (IJSR), Volume 4 Issue 3, March (2015).

[2] I. Arokiarani, R. Dhavaseelan, S. Jafari, M. Parimala, "On Some New Notions and Functions in Neutrosophic Topological Spaces", Neutrosophic Sets and Systems, Vol. 16, (2017).

[3] K. Atanassov "Intuitionistic fuzzy sets", Fuzzy Sets and Systems 87-94, 20(1986).

[4] Blessie Rebecca.S, A.Francina Shalini, "NEUTROSOPHIC GENERALIZED REGULAR SETS IN NEUTROSOPHIC TOPOLOGICAL SPACES", IJRAR February 2019, Volume 6, Issue 1, 2019.

[5] Blessie Rebecca.S, A.Francina halini, "NEUTROSOPHIC GENERALIZED REGULAR CONTINUOUS FUNCTION IN NEUTROSOPHIC TOPOLOGICAL SPACES",IJRAR February 2019, Volume 6, Issue 1,2019

[6] C.L. Chang, "Fuzzy Topological Spaces", J.Math. Anal ,Appl , 182-190,24(1968).

[7] Coker, D., "An introduction to intuitionistic fuzzy topological spaces", Fuzzy sets and systems, 8189,88 (1997).

[8] R.Dhavaseelan ans S.Jafari, "Generalized Neutrosophic closed sets", New trends in neutrosophic theory and applications Volume
II,261-273,(2018).

[9] R. Dhavaseelan, S. Jafari and Md. Hanif Page, "Neutrosophic generalized $\alpha$-contracontinuity”,CREAT. MATH. INFORM ,No. 2, 133 - 139,27 (2018).

[10] R. Dhavaseelan, E. Roja and M.K. Uma ,'Generalized Intuitionistic Fuzzy Closed sets", Advances in Fuzzy Mathematics ISSN 0973533X Volume 5, pp. 157-172,Number 2(2010).

[11] Dhavaseelan, R., Roja, E. and Uma, M. K., "Generalized intuitionistic fuzzy contracontinuous functions", The Journal of Fuzzy Mathematics, No. 4, 1-16,20 (2012).

[12] Dontchev,T.,"Contra continuous functions and strongly S-closed spaces",InternationalJ.MathSci.,No.2, 303-310 ,19(1996).

[13] Florentin Smarandache , "Neutrosophic and Neutrosophic Logic" ,First International Conference on Neutrosophic , Neutrosophic Logic, Set, Probability, and Statistics University ofNew Mexico, Gallup, NM87301, USA(2002).

[14] Florentin Smarandache, "Neutrosophic Set - A Generalization of the Intuitionistic Fuzzy Set", University of New Mexico, Gallup, NM 87301, USA.

[15] A.A. Salama and S.A. Alblowi, "Generalized Neutrosophic Set and Generalized Neutrosophic Topological Spaces", Journal computer Sci. Engineering, Vol.(2) No.(7)(2012).

[16] A.A.Salama and S.A.Alblowi, "Neutrosophic set and Neutrosophic topological space",SOR. mathematics, Vol.(3),Issue(4),pp-31-35,(2012).

[17] A. A. Salama, Florentin Smarandache and Valeri Kromov, "Neutrosophic Closed Set and Neutrosophic Continuous Functions", Neutrosophic Sets and Systems, Vol. 4,(2014).

[18] V.K.Shanthi, S.Chandrasekar, K.Safina Begam , "Neutrosophic Generalized Semi Closed Sets In Neutrosophic Topological Spaces", International Journal of Research in Advent Technology, Vol.6, No.7, July (2018).

[19] L.A. Zadeh, "Fuzzy Sets", Inform and Control 8, 338- 353,(1965). 